\title{
Netflix, estrategia y gestión de marca en torno a la relevancia de los contenidos
}

\author{
Netflix, strategy and brand management \\ around the relevance of the content
}

Santiago Mayorga Escalada Universidad Europea Miguel de Cervantes

\section{Referencia de este artículo:}

Mayorga Escalada, Santiago (2019). Netflix, estrategia y gestión de marca en torno a la relevancia de los contenidos. adComunica. Revista Científica de Estrategias, Tendencias e Innovación en Comunicación, (18), 219-244. DOI: http://dx.doi. org/10.6035/2174-0992.2019.18.11

\section{Palabras clave}

Netflix; Branding; Gestión de marca; Engagement; Streaming; Contenido de marca

\section{Keywords}

Netflix; Branding; Brand management; Engagement; Streaming; Branded content

\section{Resumen}

Las marcas han sabido adaptarse a cada realidad determinada a lo largo de su evolución histórica. Su identidad, simbólica e intangible, ha ganado protagonismo frente a los productos y sus beneficios puramente funcionales. La llegada del siglo XXI trae consigo una serie de cambios disruptivos muy profundos: democratización tecnológica, realidad global hiperconectada, sociedad líquida, microsegmentación, etc. Este hecho deviene en un evidente cambio paradigmático que empodera tanto a los usuarios como a las marcas, situándolas en una relación de iguales donde el 
ámbito digital condiciona las principales relaciones de comunicación. Las marcas avanzan en la profesionalización de su proceso estratégico e integral de gestión, ofreciendo contenidos relevantes que les ayudan a crear experiencias únicas a través de un engagement potente con el usuario.

Netflix es una de las marcas que mayor notoriedad global ha adquirido en los últimos años a raíz de su política estratégica, volumen de negocio, política de comunicación, y oferta de contenidos. Esta cuestión valida la pertinencia de la presente investigación centrada en analizar, a través de un desarrollo teórico previo de la disciplina y su posterior adaptación a un estudio de caso, los principales elementos que dan forma a la política de gestión estratégica de marca llevada a cabo por esta plataforma de entretenimiento en streaming.

Teniendo en cuenta la naturaleza del estudio y, una vez analizados los principales resultados extraídos, observamos cómo los contenidos juegan un papel absolutamente esencial en toda la política de gestión integral y estratégica de la marca Netflix. El protagonismo de los contenidos en la construcción de la marca es transversal y va desde el propio concepto de negocio hasta la implementación de la estrategia a través de sus principales acciones de comunicación publicitaria y promocional.

\begin{abstract}
Brands have been able to adapt to each specific reality throughout their historical evolution. Its identity, symbolic and intangible, has taken prominence in the face of products and their purely functional benefits. The arrival of the XXIst century carries a series of very profound disruptive changes: technological democratization, hyperconnected global reality, liquid society, microsegmentation, etc. This fact becomes a clear paradigm shift that empowers both users and brands, placing them in a peer relationship where the digital environment conditions the main communication relationships. Brands advance in the professionalization of their strategic and integral management process, offering relevant content that helps them create unique experiences through a powerful engagement with the user.

Netflix is one of the brands that has gained the most global notoriety in recent years as a result of its strategic policy, business volume, communication policy and content offer. This question validates the pertinence of this research focused on analyzing, through a prior theoretical development of the discipline and its subsequent adaptation to a case study, the main elements that shape the policy of strategic brand management carried out for this platform of entertainment in streaming. Considering the nature of the study and, once analyzed the main results extracted, we observe how the contents play an absolutely essential role in the overall and strategic management policy of the Netflix brand. The protagonism of the contents in the construction of the brand is transversal and goes from the very concept of business to the implementation of the strategy through its main advertising and promotional communication actions.
\end{abstract}




\section{Autor}

Santiago Mayorga Escalada [smayorga@uemc.es] es Doctor en Comunicación por la Universidad Pontificia de Salamanca. Docente en la Universidad Europea Miguel de Cervantes. Miembro de la Asociación de Investigadores de la Comunicación y de la Red Iberoamericana de Investigadores en Publicidad. Sus líneas de investigación giran en torno a la gestión de marcas, el branding, las comunicaciones integradas de marca, las nuevas tendencias en comunicación y publicidad y la gestión de marcas en el deporte profesional. 


\section{Introducción}

Las marcas han sabido adaptarse a cada realidad determinada a lo largo de su evolución histórica (Eguizábal, 1998). Su identidad, simbólica e intangible, ha ganado protagonismo frente a los productos, y sus argumentos funcionales. La llegada del siglo XXI trae consigo una serie de cambios disruptivos muy profundos. Internet, junto con la democratización tecnológica, provoca que las sociedades desarrolladas evolucionen hacia una nueva realidad híper-conectada, transmedia, líquida, globalizada y micro-segmentada donde impera la inmediatez. Este hecho devine en un evidente cambio paradigmático (comunicación, comportamientos, consumo, etc.) que empodera a los usuarios, convirtiéndolos en el centro indiscutible de la acción de las marcas (Ballester y Sabiote, 2011).

Se avanza hacia una mayor profesionalización del proceso de gestión de marca, buscando mayor valor añadido y una capacidad eficiente de adaptación a la nueva realidad paradigmática. Las marcas intentan crear un engagement potente a través del establecimiento de conexiones relevantes que ayudan a crear experiencias de usuario únicas (Alloza, 2010). Se convierten el activo más valioso con que cuentan las corporaciones en la actualidad (Clifton, 2009).

$\mathrm{Al}$ calor de estas circunstancias se produce una expansión constante de la industria del entretenimiento o showbusiness. Nos encontramos ante un sector que crece exponencialmente a lo largo de las últimas décadas del siglo XX hasta llegar a ser enormemente potente en pleno siglo XXI (Bustamante, 2009). El sector audiovisual, entendido en toda su magnitud, es uno de los tentáculos más reconocibles que forman parte de la industria del entretenimiento. También lo hacen las nuevas ideas de negocio que aparecen en torno a la oferta transmedia de múltiples contenidos audiovisuales. De entre todo este espectro de nuevas ofertas destaca el caso paradigmático de Netflix.

\section{Hipótesis y Metodología}

Teniendo en cuenta lo expuesto previamente vamos a acotar de forma teórica la disciplina de la gestión de marca teniendo en cuenta los cambios disruptivos acaecidos en los últimos tiempos, así como la necesaria adaptación del proceso a la nueva realidad paradigmática. A partir de aquí podremos acercarnos a la cuestión de forma aplicada a través del estudio de caso de la marca Netflix, atendiendo especialmente al papel que juegan los contenidos dentro del proceso de gestión de la marca. La finalidad principal de cualquier estudio de caso, como técnica empírica de naturaleza cualitativa, es investigar la particularidad, la unicidad, del caso singular. En síntesis, con esta definición, pasamos a plantear la hipótesis de trabajo para la presente investigación afirmando que: «dentro del proceso de gestión de la marca Netflix los contenidos se configuran como un elemento estratégico relevante».

En el transcurso de la investigación se persigue obtener un volumen de certezas suficientes como para poder validar o refutar finalmente la hipótesis. Para ello es 
necesario establecer una serie de objetivos que, teniendo en cuenta el caso concreto, se plantean dos que dan continuidad estructural y coherencia a la investigación: acotar la disciplina que da forma al proceso de gestión de marca y estudiar los elementos estratégicos más relevantes dentro del proceso de gestión de la marca Netflix. A partir de este momento queda definida la estructura interna de la investigación en torno a dos etapas perfectamente diferenciadas y, a su vez, absolutamente complementarias: cuerpo teórico y estudio de caso.

\section{Investigación}

La técnica metodológica empleada para desarrollar la fase asociada al primer objetivo de la investigación es la revisión bibliográfica y documental de expertos en gestiones de marca, nacionales e internacionales, procedentes tanto del sector profesional como del ámbito académico.

\subsection{Cuerpo teórico: gestión de marca}

Nos acercamos al estudio del proceso de gestión de marca a través de las Ciencias de la Comunicación. La disciplina se conforma a lo largo del tiempo en torno a la integración de tres grandes corrientes: el management (Kapferer, 2012), el marketing (Keller, 2008) y la comunicación estratégica/comercial (De Chernatony y Lynch, 2004).

\subsubsection{Historia y evolución de las marcas}

El papel de la marca ha pasado por diferentes momentos a lo largo de la historia, adaptándose a las necesidades de las personas en cada una de las diferentes civilizaciones existentes. Su evolución más relevante se produce a finales del siglo XIX y a lo largo de todo el siglo XX (Eguizábal, 2007). En la actualidad las marcas gozan de un papel protagonista constituyéndose como el activo más relevante y valioso con el que cuentan las corporaciones.

La naturaleza histórica de las marcas ha sido siempre motivo de estudio. Nos encontramos ante un fenómeno que por su naturaleza resulta difícil de acotar (Benavides, 2013). Esta situación provoca que se realicen numerosos acercamientos a la materia a través de diferentes corrientes y disciplinas lo que genera múltiples interpretaciones. Los expertos, en vez de avanzar hacia un marco teórico claro, conciso y pedagógico tienden a construir perspectivas personalistas donde prima el afán comercial o el posicionamiento profesional (Fernández Gómez, 2013).

El papel protagonista que adopta la marca en nuestros días conlleva un enorme ejercicio de dinamismo y capacidad de innovación que facilite la adaptación constante a la coyuntura del momento (Keller, 2008). Teniendo en cuenta esta perspectiva vamos a entender la marca como la experiencia de una promesa cumplida (Mayorga, 2018). 


\subsubsection{El proceso de gestión de marcas}

La disciplina que da lugar al desarrollo de todo este proceso estratégico de gestión de una marca es enormemente compleja. Al igual que ocurre con el fenómeno de la marca, existe una confusión atávica a la hora de acercarse a esta disciplina tanto desde el punto de vista profesional como desde el ámbito académico (Fernández Gómez, 2013; Benavides, 2017). Esta problemática se reproduce de forma escalonada a la hora de identificar elementos que son inherentes al proceso. Esta cuestión conduce a que no exista un marco teórico de consenso entre especialistas.

Conforme a lo expuesto construimos una amalgama teórica de expertos para lograr acotar la disciplina de la gestión de marcas. Para ello tomamos los principales ítems que nos llevan a la construcción de un marco teórico básico. De acuerdo con esta lógica afirmamos que la gestión de marca se conforma como un proceso estratégico integral, de naturaleza multidisciplinar, que logra alinear de forma coherente todas las acciones y elementos que van a dar forma a la marca frente a la percepción de sus públicos (Keller, 2011; Ayestarán, 2016; Rosenbaum-Elliot, Percy y Pervan, 2015). La administración de un proceso tan complejo sólo puede desarrollarse con éxito si se realiza de una forma profesional y planificada (Velilla, 2010). Acotamos la naturaleza del proceso de gestión de marcas a través de las principales características que le dan forma:

Sistema holístico de naturaleza multidisciplinar

La realidad del sector nos indica que cualquier intento por comprender la disciplina tiene que escapar de la atomización y de los planteamientos reduccionistas, buscando la integración de todos los elementos que conforman el proceso (Baena y Cerviño, 2014).

Nos encontramos ante un sistema complejo, de naturaleza eminentemente multidisciplinar. Joan Costa acota la disciplina a través de su naturaleza holística donde «[...] no se puede conocer las partes sin conocer el todo, y a la inversa... el todo es más que la suma de sus partes...el todo no es posible reducirlo a partes porque todas ellas son interdependientes y es así como toman sentido al formar el todo» (Costa, 2013: 14).

Proceso estratégico de gestión integral

Si hay una característica fundamental que recorre de forma transversal el proceso holístico de gestión de marca es su carácter estratégico (Aaker y Joachimsthaler, 2005). Nos encontramos ante un sistema complejo que, a través de la estrategia, alinea de forma integral todos los elementos, acciones, agentes y disciplinas que lo componen, alineándolos en torno a un objetivo común a largo plazo (Munuera Alemán y Rodríguez Escudero, 2006). 
Adaptarse al presente, predecir el futuro

Cualquier proceso de gestión de marca ha de saber adaptarse al presente, estando prevenido para anticiparse a las condiciones futuras (Fernández Gómez, 2013). Los paradigmas clásicos han caído con la llegada del siglo XXI gracias, en gran medida, al desarrollo y la democratización tecnológica (Stalman, 2014). En este sentido la marca es el único agente poderoso desde el que las corporaciones pueden establecer conexiones potentes con los usuarios a largo plazo, ofreciéndoles experiencias únicas y conversaciones pertinentes de tú a tú.

Propuesta de valor, única y diferente

El proceso de gestión de marca busca, de forma estratégica e integral, posicionarse de una forma determinada tanto dentro del mercado en el que opera como en la mente de sus públicos objetivo (Aaker, 2012).

El fin último de la marca, a través de su proceso estratégico de gestión, es dotarse de valor añadido. La creación de una experiencia única y relevante, cumpliendo la promesa realizada, hace que los usuarios fidelicen su relación con la marca y estén dispuestos a pagar más por ella. Este es el momento ideal donde el proceso de gestión de marca alcanza su plenitud (Keller, Parameswaran y Jacob, 2011).

Coherencia, constancia y consistencia

Cualquier proceso de gestión de marca exitoso, desarrollado a través de un plan estratégico integral, reúne tres características comunes que se antojan indispensables: coherencia, constancia y consistencia (Stalman, 2014: 43).

\subsubsection{Etapas estructurales del proceso}

Recogiendo tanto la naturaleza esencial como las características básicas de la disciplina se conforma una suerte de artefacto teórico que logra acotar el fenómeno. Para finalizar este trabajo identificamos dos grandes partes que dan sentido estructural al proceso (Cerviño y Baena, 2014; De Chernatony, 2010; Healey, 2009; Ollé y Riu, 2009).

Construcción/estrategia de marca. Se refiere a la parte interna en que se da forma a la estrategia y se alinean a todos los elementos de construcción de la marca en torno a ella. Esta parte se divide a su vez en una serie de etapas de descubrimiento hasta llegar a la conformación de la idea de negocio, la identidad, el concepto y el posicionamiento de la marca a través de un concepto único y diferenciador en forma de promesa a sus públicos (Benavides, 2017).

Comunicación de marca. Esta parte hace referencia a la implementación de la estrategia de marca a través de todos los elementos y acciones de comunicación necesarios para hacer eficiente la conexión con el target (Fill y Turnbull, 2016). 
Esta parte tiene que ver tanto con elementos internos como con acciones externas de comunicación, estando coordinadas en todo momento por un programa de comunicaciones integradas de marca.

Hay otras dos etapas reconocibles dentro de todo proceso de gestión de marca. Teniendo entidad propia, son subsidiarias de la parte de construcción y comunicación de marca, activándose en función de las necesidades estratégicas del momento. Hablamos de la tarea de medición (Aaker, 2012) y de arquitectura de marca (Martín García, 2005).

\subsection{Estudio de caso: gestión de marca Netflix y relevancia de los contenidos como elemento estratégico dentro del proceso}

Para desarrollar de forma procedente esta etapa se va a utilizar una integración de tres técnicas metodológicas: revisión documental, observación de elementos y cotejo de información para identificar aspectos relevantes (Marshall y Rossman, 1989).

De forma lógica y coherente con los objetivos planteados utilizamos, como anclaje estructural, el marco teórico construido en el punto anterior. Toda vez que tenemos acotada la disciplina y, conocemos las partes fundamentales que dan forma al proceso, podemos establecer un guion lógico que aplicaremos en el análisis del proceso de gestión estratégica de la marca Netflix. Teniendo en cuenta la hipótesis y los objetivos marcados en la investigación se analiza de forma específica y transversal el papel que juegan los contenidos dentro de las dos partes fundamentales en que se divide el proceso:

\subsubsection{Construcción/estrategia de marca}

Como ya se ha desgranado en el marco teórico, la parte de construcción de marca hace referencia a la conformación interna de una determinada estrategia para la marca, estableciendo una serie de líneas maestras que seguir en el medio y largo plazo (Ávalos, 2010). A través de esta fase se conforma la personalidad, los valores, los atributos y el concepto clave que va asociado al posicionamiento, la diferenciación y la propuesta de valor de la marca (Keller, 2008). Con el objeto de ser precisos con la investigación y lograr entender la coherencia interna del proceso vamos a analizar los elementos más significativos que dan forma a esta primera parte:

\section{a. Historia}

'Netflix, Inc.' nace en el año 1997. Todo comienza cuando Reed Hastings es penalizdo económicamente por 'Blockbuster' al retrasarse en la devolución de una película. A raíz de esta circunstancia pone en marcha un modelo de negocio innovador: alquiler de películas desde tu casa sin recargos (Tucker, 2015). En 1999, Netflix lanza una oferta de suscripción mensual con posibilidad de alquiler ilimitado de películas (Thompson, 2003). Todas las ventajas competitivas quedan integradas ese mismo 
año en torno a una web. En el año 2000 la marca amplía notablemente los servicios online, introduciendo un programa informático que ofrece recomendaciones a los usuarios de forma individualizada. En 2002, Netflix cuenta con más de 600.000 usuarios. En 2003 implementan una patente informática que facilita los trámites online y ofrece un nuevo modelo de sugerencias a través de la valoración de sus propios usuarios. A lo largo del año 2006 llevan a cabo un concurso tecnológico para seguir desarrollando patentes.

El negocio de Netflix crece exponencialmente apostando por una ofertad de calidad en sus contenidos a través de un servicio online cada vez más eficiente (Tucker, 2015). En 2007 la marca revoluciona el sector introduciendo la reproducción de sus contenidos vía streaming. En 2008 y 2009 amplían su mercado, introduciéndose en plataformas televisivas y dispositivos móviles. El número de suscriptores asciende a los 10 millones en el año 2009 (Netflix Media Center, 2018).

Netflix cierra en el año 2010 su primera expansión de mercado dentro de un nicho estratégico, implantando su presencia en todos los dispositivos móviles de Apple. A partir de este momento se pone en marcha el gran objetivo: globalizar la marca. Ese mismo año se introduce en el mercado canadiense. En 2011 expande su oferta a Latinoamérica y el Caribe. A partir del año 2012 aterriza de forma escalonada en Europa, Reino Unido y países nórdicos. La expansión global continúa con éxito en el año 2013, convirtiéndose en la primera plataforma online que recibe un Primetime Emmy por una serie de producción propia, House of Cards.

En 2014 la marca cuenta con 50 millones de suscriptores. En 2015, Netflix se introduce en Australia, Nueva Zelanda y Japón. Ese mismo año cierra su desembarco en Europa con la entrada en el mercado español, italiano y portugués. En 2016 alcanza su objetivo estratégico de expansión comercial internacional y, en 2017, cuenta con más de 100 millones de suscriptores (EuropaPress, 2017).

A partir del año 2018 la marca se fija nuevos retos estratégicos: aumentar paulatinamente su inversión en contenido propio hasta llegar al 50\% de su oferta (Statt, 2017), uso integral del big data y adaptación a las diferentes realidades locales. Netflix cierra 2018 con 139 millones de suscriptores lo que supone un incremento del 35\% con respecto a los datos del año anterior. Los ingresos de la compañía subieron este pasado año un 27\%, situándose en los 4.190 millones de dólares (Netflix Investors, 2019).

Para 2019 Netflix se plantea aumentar de forma significativa sus ingresos a pesar de las inversiones en contenido propio y la amenaza de la competencia (Disney creará su propia plataforma). En este sentido los datos del primer cuatrimestre del año indican que la marca sigue creciendo aunque por debajo de los ambiciosos resultados que tenía previstos: 7,86 millones de nuevos suscriptores y un aumento de los ingresos totales que pasan a 4.520 millones de dólares frente a los 3.700 millones de dólares en el mismo cuatrimestre del pasado año (Netflix Investors, 2019). 


\section{b. Idea de negocio}

Conocer la historia de la empresa junto con sus principales ítems corporativos facilita comprender cuál es la idea central del negocio. Esta cuestión pone de manifiesto el concepto estratégico de la marca y cómo se adapta a las circunstancias a lo largo del tiempo a través de la toma de decisiones.

La marca Netflix se posiciona, según la agencia neoyorkina encargada de realizar su último gran programa de branding, como «el servicio de streaming de medios líder en el mundo [...] Uno de los innovadores originales del contenido en streaming. [...] Con una amplia gama de series originales, adquisiciones de alto perfil y una base de usuarios en explosión. [...] Netflix es una marca viva, en constante evolución» (Gretel, 2013).

De acuerdo con el posicionamiento de la marca y, teniendo en cuenta la información extraída a través del trabajo de revisión bibliográfica realizado, vamos a resumir la idea de negocio de Netflix en cinco conceptos estratégicos clave:

- Marca global. Netflix pone en marcha diferentes planes de crecimiento internacional de su marca a partir del año 2010, dando por concluida la fase de expansión global en el año 2018. Este trabajo se desarrolla de forma planificada, a través de diferentes etapas, y entendido por la compañía como una de las principales líneas maestras de su estrategia de negocio (IzquierdoCastillo, 2015). Los contenidos son contratados o producidos teniendo en cuenta el mercado global en el que opera, abriendo el abanico de ofertas a los contenidos de tipo local una vez que la marca se ha asentado a lo largo de muy diferentes países.

- Desarrollo tecnológico. Netflix ha logrado adaptarse a la nueva realidad paradigmática, entendiendo los cambios disruptivos que se producen y ofreciendo soluciones tecnológicas relevantes al servicio de las demandas de consumo y comunicación que exigen los usuarios. La marca hace suya la filosofía de aprender para adaptarse (Chahal, 2017). Todos los desarrollos tecnológicos que logran implantar vienen precedidos de un proceso de investigación, desarrollo, innovación y soluciones concretas a cuestiones determinadas que han sido perfectamente identificadas previamente. La innovación tecnológica de Netflix se convierte en una parte imprescindible de su propuesta de negocio, siempre al servicio del usuario, siendo especialmente relevante a través de tres soluciones estratégicas:

- Streaming. Netflix entiende que el usuario se sitúa en el centro de las acciones de la marca con el fin de satisfacer sus principales necesidades. En este sentido revoluciona la industria audiovisual y del entretenimiento dándole la posibilidad al usuario de consumir cualquier contenido que se oferta dentro de la plataforma en el momento que quiera, desde el lugar que quiera y a través de la pantalla/dispositivo que más le apetezca (Summers, Brecht, Eager y Gutarin, 2016). Todo ello es posible a través de la reproduc- 
ción de contenidos por streaming. Esta decisión estratégica sitúa a Netflix como una marca líder, puntera en el ámbito de la innovación tecnológica, que logra reinventar el sector. En este sentido algunos académicos sitúan a Netflix como una marca pionera dentro de la denominada nueva «industria del saber», directamente ligada con la innovación tecnológica en el ámbito digital (Burroughs, 2019).

- Convergencia y mutación. Netflix logra hacer realidad su principal promesa de marca a través de una plataforma online donde el consumidor tiene la posibilidad de acceder vía streaming a los mejores contenidos del mercado. Se conforma un sistema de consumo audiovisual online de naturaleza convergente que enlaza con los modelos teóricos descritos a comienzos de la década de los años noventa (Scolari, et al, 2012). El desafío que lanza Netflix al sector audiovisual del entretenimiento va más allá y, en este aspecto, logra conformar una oferta integral que aúna, siempre en beneficio del consumidor, la fórmula de: internet + cine + televisión (Heredia, 2017). Netflix genera un engagement muy potente al conectar de forma relevante la marca con su público a través de un sistema de convergencia audiovisual que cuenta con la mejor oferta de contenidos. En definitiva, nos encontramos ante un nuevo modelo que logra mutar la oferta de contenidos audiovisual de entretenimiento vía streaming (Ahumada, 2013).

- Gestión de información y datos de usuario. La apuesta por el desarrollo tecnológico con el fin de ofrecer un servicio totalmente adaptado a las necesidades de sus usuarios ha llevado a Netflix a buscar, a lo largo de su existencia, la mejor forma de manejar datos y recoger el feedback de usuario. La demanda del usuario al servicio del diseño de la oferta como pata estratégica de la marca (Fernández-Manzano, Neira y Clares-Gavilán, 2016). Este hecho ha sido determinante para disponer de una oferta absolutamente microsegmentada e individualizada gracias a las inversiones en big data, hasta llegar al actual algoritmo con el que trabaja la marca en el que se incluye el uso de inteligencia artificial (Carrillo, 2019). Tod Yellin, vicepresidente de producto de Netflix afirma que: «No hay nada complicado en el algoritmo de Netflix. Es realmente una colaboración entre las personas que ven Netflix, nuestros usuarios, y nosotros, tratando de hacer más fácil el camino para que alguien encuentre el contenido correcto en el momento indicado» (Schulkin, 2019). La toma de decisiones a través del uso de big data en Netflix garantiza la eficiencia de resultados en todos sus ámbitos: inversión en contenido externo, tipología temática de los contenidos, producción propia de contenidos, marketing, etc.

- Calidad y contenidos propios. La marca canaliza su éxito a través de una apuesta estratégica por invertir en la compra y/o la producción de contenidos de calidad que resulten de interés para sus usuarios. Netflix nace como un mero distribuidor para pasar a convertirse en productor de sus propios 
contenidos (2017). En el año 2013 se estrena en la plataforma su primera serie de producción propia, House of cards, y dos años después hace lo propio en formato película con Beast of no Nation, estrenada en este caso de forma simultánea en la plataforma y en el circuito habitual de cines. A partir de estos hitos la marca establece de forma estratégica en 2016 una serie de objetivos centrados en aumentar los contenidos de producción propia por encima del $50 \%$ de la oferta total, llegando en una segunda etapa que comienza a finales del año 2017 a apostar por la producción propia adaptada al ámbito local/nacional (Fernández, 2019).

\subsubsection{Comunicación de marca}

En coherencia con lo expuesto en el marco teórico y, una vez analizados los elementos principales que dan forma a la parte de construcción, vamos a describir los principales elementos que cimentan la parte de comunicación de la marca Netflix, y cuál es el papel que juegan los contenidos dentro de la misma.

La agencia global de branding y comunicación estratégica 'Gretel' se encarga de implementar el posicionamiento estratégico para conectarlo con los públicos de interés a través de diferentes técnicas de comunicación en múltiples puntos de contacto. De esta manera vamos a analizar los elementos clave que van a guiar la estrategia de comunicación de la marca:

\section{Unificación de la marca}

Netflix logra experimentar en los últimos años un crecimiento sobresaliente gracias al desarrollo de nuevos objetivos de negocio, manteniendo en todo momento la coherencia de su concepto estratégico. Naz Arandi, planner de la agencia 'Gretel', expone que la estrategia de unificación realizada para Netflix se desarrolla a través de un consumer insight. La promesa de la marca se revela de la siguiente forma: 'conectamos a la gente con historias para amar' (Arandi, 2015). Este insight es el epicentro sobre el que se cimenta toda la estrategia de comunicación de la marca. Es evidente el papel absolutamente relevante que se le da desde Netflix a los contenidos (historias para amar), siendo la principal razón de comunicación (promesa de marca/propuesta de valor) para lograr conectar con su target.

\section{Programa de identidad visual}

De forma coherente con la estrategia se desarrolla todo un sistema visual encargado de visibilizar la identidad de la marca. Esta comunicación gráfica va a servir además de plataforma para incluir y promocionar de forma protagonista los contenidos más destacados que ofrece la marca. La agencia 'Gretel' señalada que: 
«nuestro reto era crear algo lo suficientemente amplio para una marca global pero que a la vez fuese único e identificable. Crear algo flexible pero a la vez esquemático y fuerte. Tenía que ser visualmente llamativo, adaptable a cada formato e interpretable por las agencias y distribuidores de todo el mundo» (Diligent Team, 2015).

El programa de identidad visual va a girar en torno a «The Stack» (Vargas, 2015): una metáfora visual y un sistema gráfico de identidad en uno; es un catálogo de exposición de contenidos dinámico e infinito. El look y feel de la marca va a girar en torno a esta construcción gráfica donde los contenidos, como vemos, son un elemento estratégico fundamental de comunicación.

Figura 1. Uso implementado de la construcción de identidad visual «The Stack»

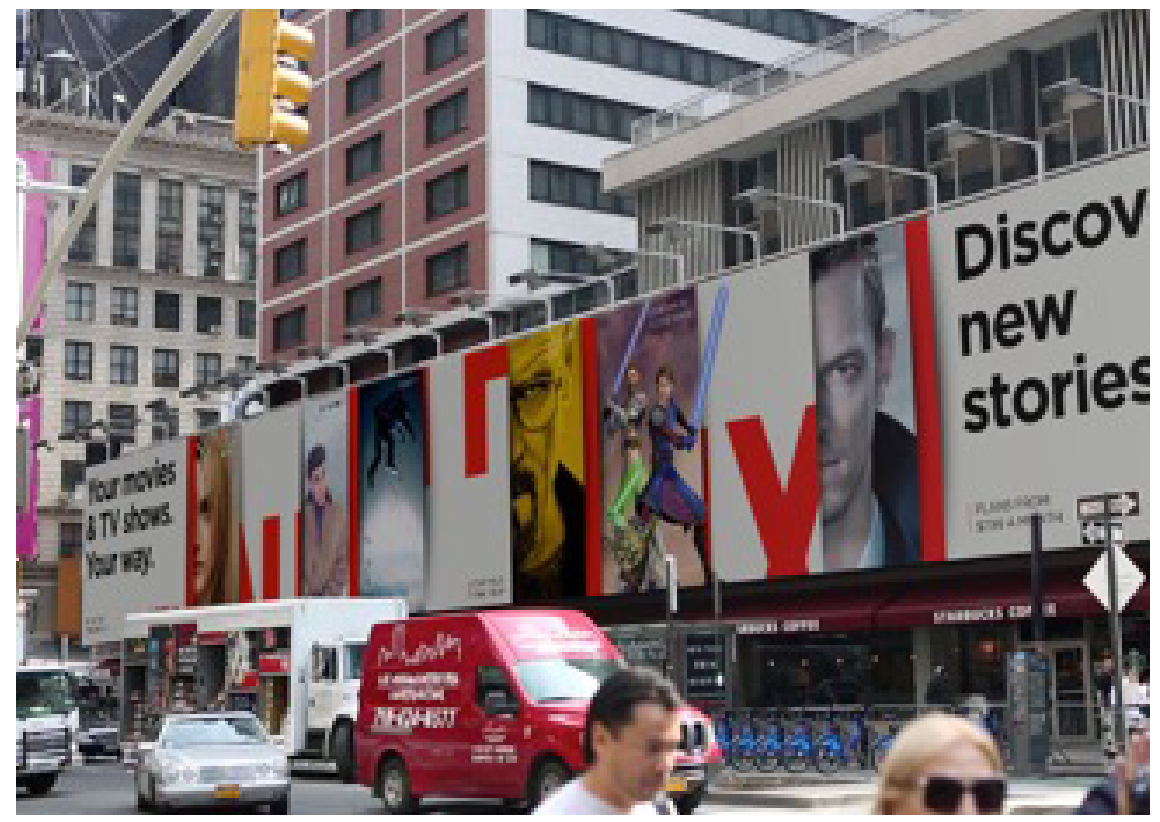

Fuente: Netflix.

Junto al programa de identidad visual que comunica de forma gráfica el concepto de la marca se produce un restyling del logo para que también quede alineado con la estrategia. Nos encontramos ante una construcción visual, compacta y minimalista, que se lee con mayor facilidad lo que permite que sea usado con fondos que hagan alusión a la oferta de contenidos de la plataforma. Netflix presenta en 2016 una versión reducida del logo con el fin de adaptarse y facilitar su implementación en diferentes situaciones del entorno digital, representando un significado simbólico 
de la continuidad en la oferta de contenidos de calidad (García, 2016). Este último logo también puede entenderse como un guiño a los contenidos en lo temporal: pasado (cinta de las películas) y presente (binge-watching).

Figura 2. Logo y logo reducido para el entorno digital

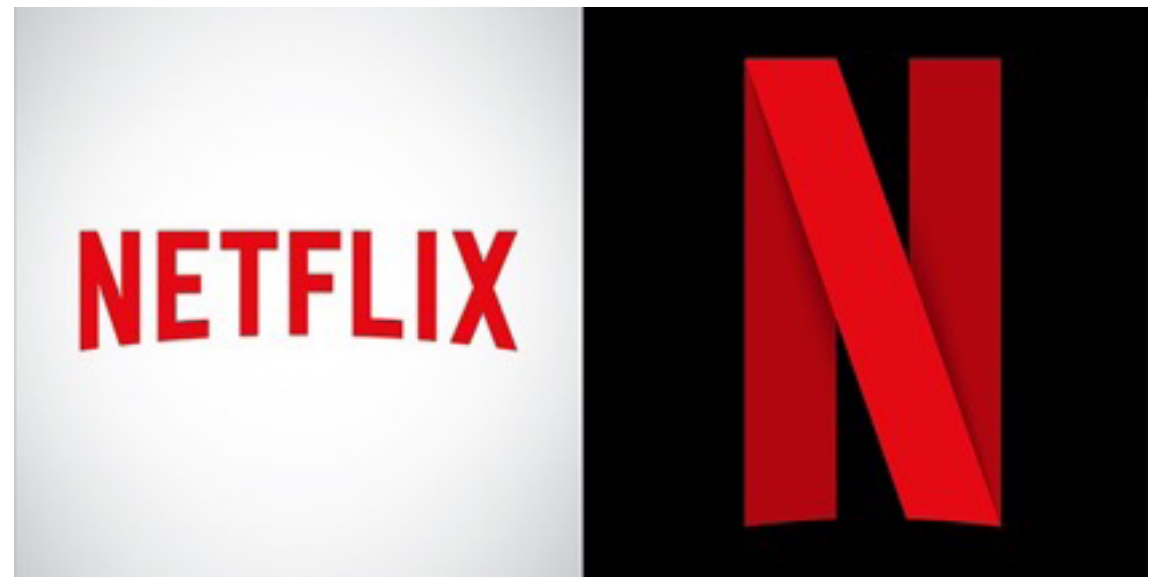

Fuente: Netflix.

Nos encontramos ante otro elemento estratégico de comunicación protagonista, encargado de dar sentido global al concepto de la marca en todos sus puntos de contacto con los usuarios. Con base nuevamente en el insight consumer, la agencia Gretel establece como global brand claim el: «See what's next». En torno a esta frase se filtra de forma implícita la oportunidad que se le ofrece al usuario de descubrir constantemente nuevas historias a través de la enorme oferta de contenidos de calidad de que dispone la marca, creando engagement gracias a una experiencia única. Es una construcción que además potencia la política de marca tanto en el ámbito de la innovación tecnológica como en su dimensión global.

El brand claim es desmenuzado por la agencia Gretel de forma estratégica, aludiendo a las siguientes asociaciones de conceptos donde queda manifiestamente claro el protagonismo que se le da a los contenidos (Whipps, 2016): «See: Connecting people with stories, Looking fordward, Exploring, Viewing the content. Next: What will Netfilx do next?, Innovation, Anticipation, Curiosity, The next show/episode». 
Figura 3. Brand claim de Netflix y desarrollo estratégico de conceptos asociados a la marca
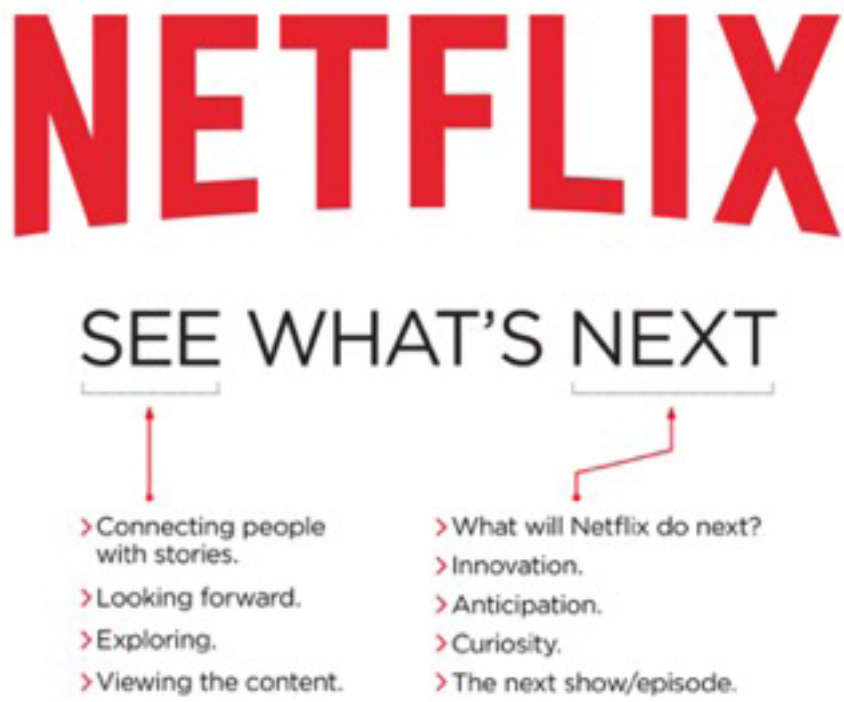

Fuente: Whipps (2016).

Netflix ha ido construyendo y utilizando toda una serie de claims alternativos que, de forma coherente con el brand claim principal, mantienen la comunicación del concepto y de los atributos sobre los que se ancla la marca. Algunos de estos claims han sido: «Anytime. Anywhere. Instantly»; «All episodes, all seasons, now streaming»; «Your movies y TV shows. You way». Es evidente cómo los contenidos (propios y de calidad) juegan un papel protagonista y transversal en los claims junto con las otras dos patas estratégicas de la marca: desarrollo tecnológico y marca global. Las fortalezas estratégicas de la marca puestas al servicio de los usuarios.

Comunicaciones integradas de marca

La gestión del resto de elementos que intervienen en la parte de comunicación se implementa a través de la elaboración de un programa de comunicaciones integradas de marca donde destaca el trabajo eficiente de marketing basado en el uso de big data, el feedback de usuario a través de redes sociales, el relato uniforma y coherente a través del trabajo de storytelling, y el branded content donde los contenidos propios de la plataforma retroalimentan las promociones de la marca. Netflix 
logra comunicar con éxito, y de forma integral-transmedia, su concepto de marca a través de potenciar sus fortalezas estratégicas.

De forma general, la marca desarrolla una inversión en marketing centrada en fijar tanto su posición global como los atributos principales que dan sentido a la promesa de negocio, adaptándose a los mercados locales con campañas publicitarias y acciones de comunicación concretas que buscan el impacto y la viralización, donde los contenidos de la plataforma son los principales protagonistas (Miguel, 2017).

Figura 4. Promo publicitaria de la serie Narcos en la Puerta del Sol de Madrid

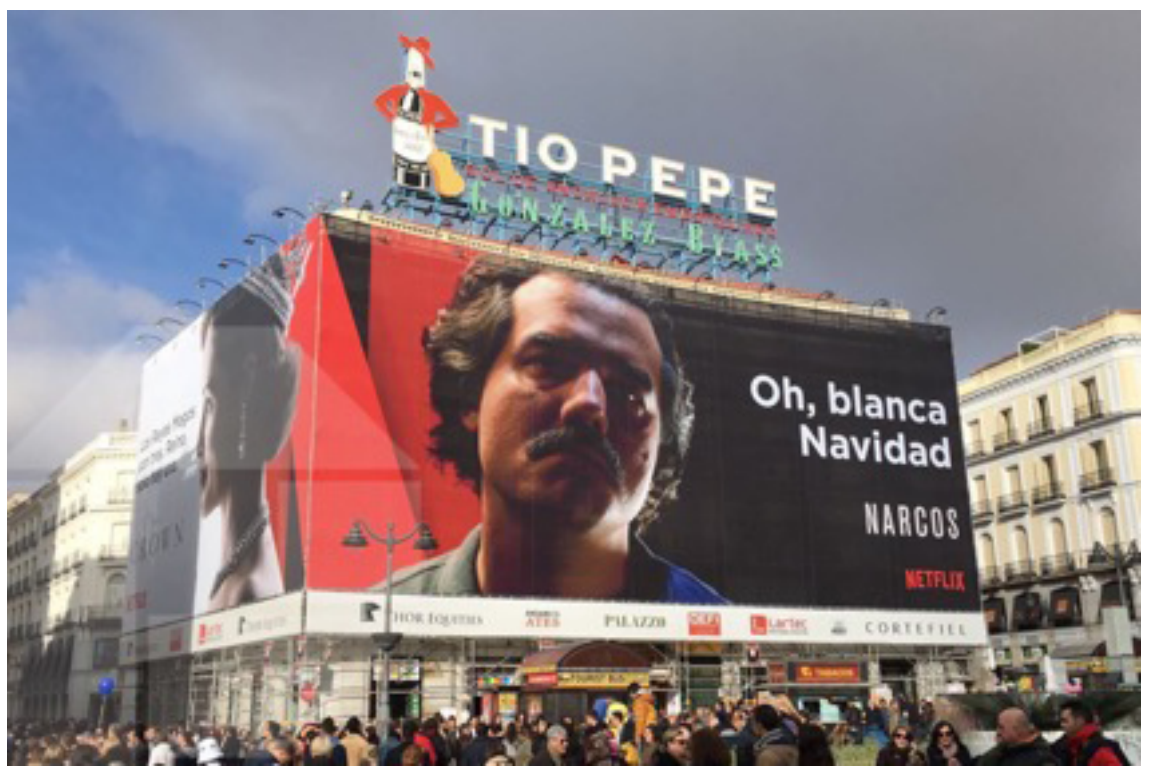

Fuente: Netflix.

El uso que hace Netflix de las redes sociales es muy activo y dinámico, cercano con el usuario, utilizando un tono desenfadado donde el humor conecta de forma potente con su target a través de la activación de conversaciones de tú a tú. Las autopromociones de contenido son las que más tráfico registran en redes y las que más actividad de usuarios generan. En todas las acciones de comunicación comercial de la marca, reconocidas por ser originales e impactantes, aparece de forma protagonista el contenido que ofrece Netflix a modo de un gran catálogo de experiencias. Resulta realmente llamativo el juego de comunicación que hace la marca en redes sociales con sus usuarios a través del uso de múltiples gifs (todos ellos extraídos de series propias que se ofertan en la plataforma). 
Figura 5. Comunicado en redes sociales de Netflix España a sus usuarios el día 1 de enero

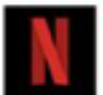

\section{Netflix}

1 de enero a las 12:07 - Madrid, Comunidad de Madrid - $\Theta$

\section{Atención: Hoy estamos con ibuprofeno, mucha agua y las luces bajitas. Ponte alguna serie mientras volvemos.}

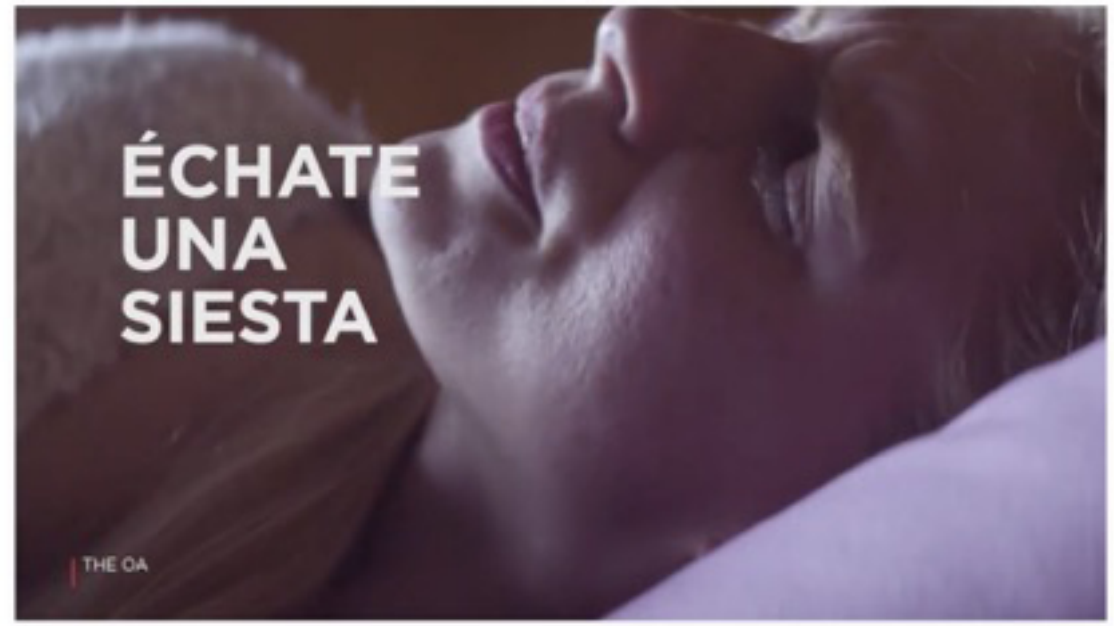

\section{6 reproducciones}

Fuente: elaboración propia.

El principal baluarte en las comunicaciones integradas de marca dentro del caso de Netflix es el marketing de contenidos, o branded content. Se utilizan los contenidos que ofrece la plataforma en todo tipo de acciones de comunicación no intrusivas con el objetivo de conectar de forma relevante con el target. De esta forma se logra afianzar el awareness y la imagen de la marca, además de activar la promesa de una experiencia única. Artículos en prensa, movimientos sociales virales, notoriedad a historias y personajes reales, apps, cameos, autoparodias, eventos o promociones cargadas de polémica son algunos de los principales ejemplos que ilustran el abanico de acciones de marketing de contenido exitoso desarrollado por Netflix. 
Figura 6. Campaña de promoción en Alemania de la serie Santa Clarita Diet

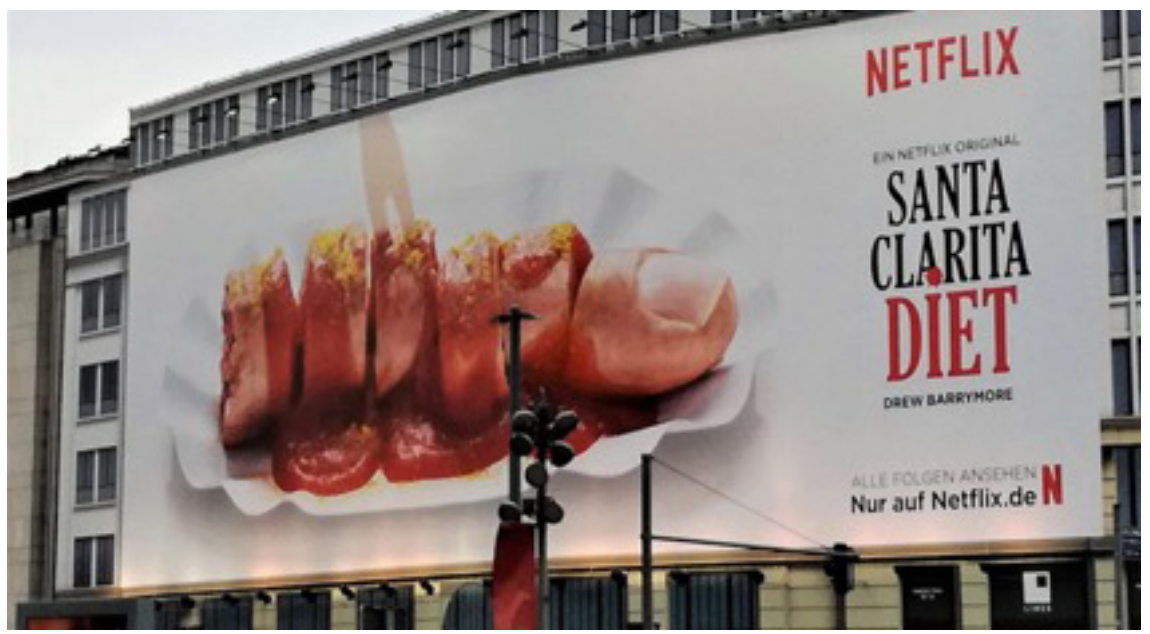

Fuente: Netflix.

Tener el concepto de la marca perfectamente definido y utilizarlo como elemento estratégico a la hora de comunicar permite que Netflix maneje con total naturalidad todos los campos de batalla de la comunicación estratégica, integrando perfectamente tanto la dimensión publicitaria/promocional online como la offline. La estrategia y la creatividad en la comunicación dan valor a las acciones que posteriormente pueden ser desplegadas a través de diferentes soportes. Pasamos de las campañas publicitarias gráficas o audiovisuales más tradicionales a los gifs y el lenguaje de redes sociales, manteniendo en cualquier caso el mensaje sobre el protagonismo de un contenido determinado que se oferta en la plataforma. Pero Netflix va mucho más allá y ha desarrollado campañas de marketing y comunicación innovadoras en el ámbito de la realidad virtual $360^{\circ}$ (Stranger Things), la cocreación junto con otra marca como Google (máquina expendedora que se controla con la mente. Stranger Things), la tecnología face swap (House of cards), las apps (entrenador personal con personajes de series, gifs de sus series, de servicios en temas relacionados con los contenidos que oferta, etc.), inventos para potenciar la experiencia de usuario (los calcetines que pausan tu serie si te duermes, construir el botón de Netflix para automatiza algunas de las tareas necesarias para un agradable visionado de series, etc.) y la producción propia de promociones basadas en la utilización de elementos genuinos de la marca (contar una historia a través del concepto binge watching para la campaña Binge for love de San Valentín). 
Figura 7. Justificante laboral de Netflix para usuarios que han estado practicando binge la noche anterior
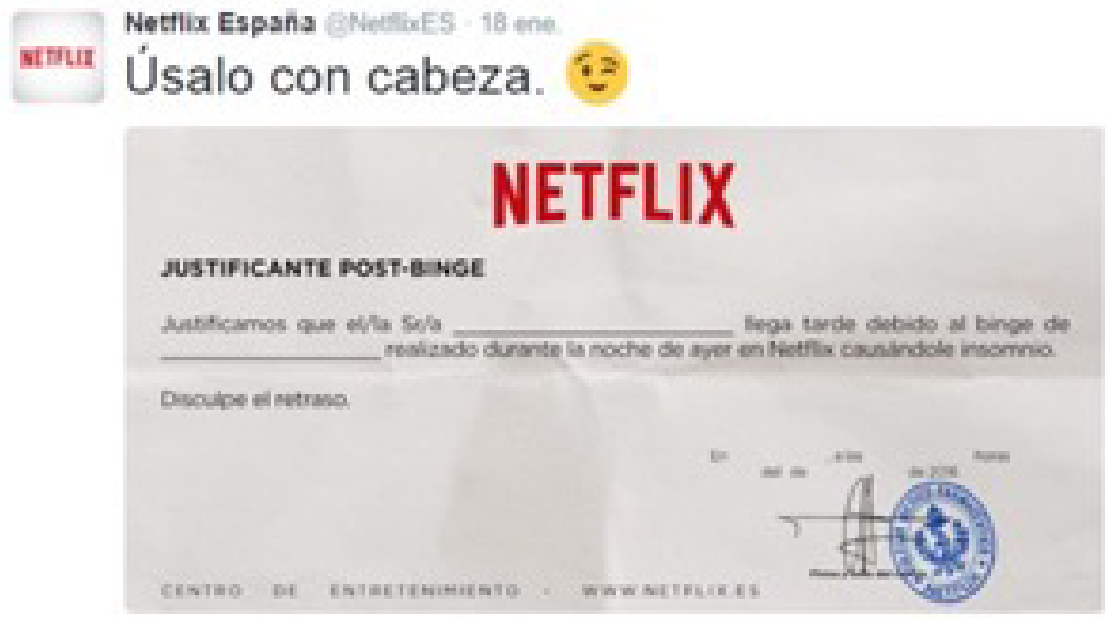

Fuente: elaboración propia.

Los planes de marketing y comunicaciones integradas de marketing son otro punto estratégico de la marca para la toma de decisiones eficaces a través del uso de avances tecnológicos: big data y data management. En este caso el algoritmo de Netflix recoge una serie de información y variables de usuario que ayudan a establecer una tipología determinada a la hora de segmentar para plantear posteriormente acciones concretas de marketing en torno a al protagonismo de los contenidos.

\section{Conclusiones}

Después de realizar el proceso de investigación se han extraído una serie de datos que, tras ser analizados, vamos a presentar de forma ordenada como conclusiones que nos llevarán a dar una respuesta escalonada tanto a los objetivos como a la hipótesis planteada.

Objetivo 1. Acotar la disciplina que da forma al proceso de gestión de marca.

Teniendo en cuenta la problemática atávica existente en torno a la gestión de marcas para lograr un consenso entre expertos que acote la disciplina se crea un cuerpo teórico ad hoc a través de la revisión bibliográfica donde: 
- Se define la gestión de marcas como un proceso estratégico integral, de naturaleza multidisciplinar, que logra alinear de forma coherente todas las acciones y elementos que van a dar forma a la marca frente a la percepción de sus públicos.

- Las características principales que dan forma al proceso de gestión de una marca son: sistema holístico de naturaleza multidisciplinar, proceso estratégico de gestión integral, adaptación al presente y predicción del futuro, propuesta de valor única y diferente de forma coherente, constante y consistente.

- El proceso de gestión estratégica de marca se divide en dos grandes partes: construcción (descubrimiento interno y planteamiento de la estrategia) y comunicación (implementación de la estrategia para contactar la marca con los públicos). Existen otras dos etapas que funcionan de forma subsidiaria a las principales: medición y arquitectura.

Objetivo 2. Estudiar los elementos estratégicos más relevantes dentro del proceso de gestión de la marca Netflix.

En este sentido es imprescindible remarcar la importancia de estudiar los contenidos, y cuál es su papel, dentro de las partes y elementos de constitución en que se divide el proceso.

\section{Construcción de marca}

- La historia de Netflix nos presenta una marca proactiva, dinámica y revolucionaria que pasa de retadora a ser líder global reinventando prácticamente el sector.

- El posicionamiento estratégico de la marca (los mejores contenidos al servicio de los usuarios) y su propuesta de valor pivotan en torno a tres líneas fundamentales: marca global, desarrollo tecnológico (streaming, convergencia de medios en torno a una plataforma de marca online, y gestión de información a través del big data y la conformación de un algoritmo propio) y protagonismo absoluto de los contenidos (de calidad y aumento notable de la producción propia).

\section{Comunicación de marca}

- La estrategia y el concepto conectan con los usuarios a través de una experiencia única en torno a la comunicación de un insight de consumidor que coloca a los contenidos como la pieza fundamental de la promesa de marca. Se utilizan claims complementarios que están perfectamente alineados con la estrategia de comunicación descrita. 
- El trabajo de identidad y comunicación visual gira en torno al protagonismo de los contenidos (ya sea como elementos complementarios o como principales protagonistas dentro de la construcción gráfica).

- El programa de marketing y comunicaciones integradas de marca se adapta perfectamente a la nueva realidad paradigmática, conectando de forma potente con los usuarios a través de nuevas tendencias en comunicación publicitaria online y offline. En todos los casos, los contenidos de la marca son los principales protagonistas en sus comunicaciones.

- La tecnología y el uso del big data se ponen también al servicio de la toma de decisiones en marketing y comunicación. Avala la eficacia en el protagonismo indiscutible de los contenidos.

Hipótesis. Dentro del proceso de gestión de la marca Netflix los contenidos se configuran como un elemento estratégico relevante.

Cumplir con los objetivos de la investigación nos proporciona una serie de argumentos que, tras ser pertinentemente analizados, van a llevarnos hasta la resolución de la hipótesis.

Afirmamos que los contenidos se conforman como un elemento completamente transversal dentro de la estrategia de la marca Netflix. Están presentes a lo largo de toda la historia de la compañía, jugando siempre un papel esencial tanto en la propuesta de valor como en la promesa de la marca.

Dentro del proceso de gestión de la marca los contenidos desempeñan un rol protagonista, siendo absolutamente omnipresentes. Aparecen de forma nuclear a lo largo de todos los elementos que dan forma a la parte de construcción de la marca. En la parte de comunicación se configuran como el elemento primordial que pone en contacto a la marca con los usuarios a través de la promesa de una experiencia única, generando un engagement muy potente. Tanto es así que Netflix es la marca más valorada por los millenials (Merca2.es, 2017).

Concluimos, por tanto, que la hipótesis de trabajo plateada queda totalmente validada ya que los contenidos se configuran como un elemento estratégico absolutamente relevante dentro del proceso de gestión de la marca Netflix.

\section{Referencias}

Aaker, David (2012). Building strong brands. Nueva York: Simon and Schuster.

Aaker, David y Joachimsthaler, Erich (2005). Liderazgo de marca. Bilbao: Deusto. Ahumada, Rafael (2013). La televisión por Internet: de la convergencia a la mutación. En: Quórum Académico, Vol. 10, nº 2. Valencia: Universitat de València, 277-288. 
Munuera Alemán, José Luis y Rodríguez Escudero, Ana Isabel (2007). Estrategias de marketing: un enfoque basado en el proceso de dirección. Madrid: Esic.

Alloza, Ángel (2010). Brand engagement: Marca, experiencia. En: Capriotti, P. y Schulze, F. (eds.), Responsabilidad Social Empresarial,. Barcelona: Colección Libros de la Empresa.

Arandi, Naz (2015). Netflix brand strategy + website. En: http://www.itsnaz.com/ brandstrategy/ (Consultado el 18-07-2019).

Ávalos, Carlos (2010). La marca: identidad y estrategia. Buenos Aires: La Crujía.

Ayestarán, Raquel (2016). Planificación estratégica y gestión de la publicidad. Conectando con el consumidor. Madrid: ESIC.

Ballester, Elena y Sabiote, Estela (2011). Las experiencias de marca: estudio exploratorio de sus consecuencias en la relación consumidor-marca. En: European Journal of Management and Business Economics, Vol. 20, n ${ }^{0}$ 3. Ámsterdam: Elsevier, 121-139.

Benavides, Juan (2017). Los nuevos caminos en la evolución de las marcas. Madrid: Fragua.

Benavides, Juan (2013). A propósito del Brand Management. En: Fernández Gómez, J. (ed.). Estrategia publicitaria y gestión de marcas. Madrid: McGraw-Hill.

Burroughs, Benjamín (2019). House of Netflix: Streaming media and digital lore. En: Popular Communication, Vol. 17, $\mathrm{n}^{0}$ 1. Mahwah: Lawrence Erlbaum Associates, 1-17.

Bustamante, Enrique (2009). De las industrias culturales al entretenimiento. La creatividad, la innovación... Viejos y nuevos señuelos para la investigación de la cultura. En: Diálogos de la Comunicación, Vol. 78. Lima: FELAFACS, 1-25.

Carrillo, Javier (2019). Paradigma Netflix. El entretenimiento de algoritmo. Barcelona: UOC.

Cerviño, Julio y Baena, Verónica (2014). Nuevas dimensiones y problemáticas en el ámbito de la creación y gestión de marcas. En: Cuadernos de Estudios Empresariales, $\mathrm{n}^{\mathrm{o}}$ 24. Madrid: Universidad Complutense de Madrid, 11-50.

Chahal, Mindi (2017). Netflix CEO: Focus more on stories and less on screen size. En: https://www.marketingweek.com/2017/02/28/netflix-mobile-world-congress/ (Consultado el 18-07-2019).

Fernández-Manzano, Eva-Patricia; Neira, Elena y Clares-Gavilán, Judith (2016). Gestión de datos en el negocio audiovisual: Netflix como estudio de caso. En: El profesional de la información, Vol. 4, no 25. Barcelona: El Profesional de la Información, 568-577.

Clifton, Rita (2009). Brands and branding. New York: John Wiley \& Sons. 
Costa, Joan (2013). Los 5 pilares del branding. Anatomía de la marca. Barcelona: CPC Editor.

De Chernatony, Leslie y Lynch, Joanne (2004). The power of emotion: Brand communication in business-to-business markets. En: The Journal of Brand Management, Vol. 11, no 5. Londres: Henry Stewart, 403-419.

De Chernatony, Leslie (2010). Creating powerful brands. Oxford: Routledge.

Diligent Team (2015). Unificación de marca para Netflix global. En: https://www. diligent.es/unificacion-de-marca-netflix/ (Consultado el 18-07-2019).

Eguizábal, Raúl (1998). Historia de la publicidad. Madrid: Eresma y Celeste.

Eguizábal, Raúl (2007). Teoría de la publicidad. Madrid: Cátedra.

EuropaPress (2017). Netflix supera los 100 millones de usuarios. En: http://www. europapress.es/tv/noticia-netflix-supera-100-millones-usuarios-20170718151127.html (Consultado el 18-07-2019).

Fernández, Javier G. (2019). ¿Por qué Netflix necesita más contenido original? En: http://www.expansion.com/economiadigital/companias/2019/05/05/5cc8040d2260 1de1188b45e7.html (Consultado el 18-07-2019).

Fernández Gómez, Jorge (2013). Estrategia publicitaria y gestión de marcas. Madrid: McGraw-Hill.

Fill, Chris y Turnbull, Sara Louise (2016). Marketing communications: brands, experiences and participation. Londres: Pearson.

García, Modesto (2016). Netflix presenta una nueva versión de su logo. En: http:// www.brandemia.org/netflix-presenta-una-nueva-version-de-su-logo (Consultado el 18-07-2019).

Martín García, Manuel (2005). Arquitectura de marcas: modelo general de construcción de marcas y gestión de sus activos. Madrid: ESIC Editorial.

Gretel (2013). Netflix branding. En: http://v4.gretelny.com/project/56/netflix-branding (Consultado el 18-07-2019).

Healey, Matthew (2009). ¿Qué es el branding?. Barcelona: Gustavo Gili.

Heredia, Verónica (2017). Revolución Netflix: desafíos para la industria audiovisual. En: Chasqui, Revista Latinoamericana de Comunicación, $\mathrm{n}^{\circ}$ 135. Quito: Ciespal, 275-295.

Izquierdo-Castillo, Jessica (2015). El nuevo negocio mediático liderado por Netflix: estudio del modelo y proyección en el mercado español. En: El profesional de la información, Vol. 6, n ${ }^{\circ}$ 24. Barcelona: El Profesional de la Información, 819-826.

Kapferer, Jean-Noël (2012). The new strategic brand management: Advanced insights and strategic thinking. London: Kogan page publishers. 
Keller, Kevin Lane; Parameswaran, M. G. y Jacob, Isaac (2011). Strategic brand management: Building, measuring, and managing brand equity. Londres: Pearson Education.

Keller, Kevin Lane (2008). Administración estratégica de marca. Branding. Madrid: Pearson Educación.

Marshall, Catherine y Rossman, Gretchen (1989). Designing qualitative research. Newbury: Sage.

Mayorga, Santiago (2018). La gestión de marca en el contexto universitario español: análisis de los grados de publicidad y relaciones públicas. Tesis doctoral. Salamanca: Universidad Pontificia de Salamanca.

Miguel, Esther (2017). El día en que Netflix decidió traspasar el último límite español en publicidad: ETA. En: https://magnet.xataka.com/en-diez-minutos/el-dia-enque-netflix-decidio-traspasar-el-ultimo-limite-espanol-en-publicidad-eta (Consultado el 18-07-2019).

Netflix Investors (2019). Letter to stakeholders. 2018 Quarterly Earnings. En: https://www.netflixinvestor.com/financials/quarterly-earnings/default.aspx (Consultado el 18-07-2019).

Netflix Media Center (2018). Netflix Timeline. A brief history of the company that revolutionized watching of movies and TV shows. En: https://media.netflix.com/en/ about-netflix (Consultado el 18-07-2019).

Ollé, Ramón y Riu, David (2009). El nuevo brand management: cómo plantar marcas para hacer crecer negocios. Barcelona: Gestión 2000.

Merca2.es (2017). Aviso a navegantes: Netflix, la marca del mundo más valorada por los jóvenes. En: https://www.merca2.es/netflix-marca-valor/ (Consultado el 18-07-2019).

Rosenbaum-Elliott, Richard; Percy, Larry y Pervan, Simon (2015). Strategic brand management. Oxford: Oxford University Press.

Schulkin, Julieta (2019). Claves para comenzar a entender el algoritmo secreto de Netflix. Infobae. En: https://www.infobae.com/america/tecno/2019/03/24/claves-para-comenzar-a-entender-el-algoritmo-secreto-de-netflix (Consultado el 18-07-2019).

Scolari, Carlos Alberto; Fernández de Azcárate, Sara; Garín, Manuel; Guerrero, Mar; Jiménez, Manel; Martos, Aitor; Obradors, Matilde; Oliva, Mercè; Pérez, Óliver y Pujada, Eva (2012). Narrativas transmediáticas, convergencia audiovisual y nuevas estrategias de comunicación. En: Quaderns del CAC 38, Vol. 15, $\mathrm{n}^{\mathrm{O}} 1$. Barcelona: CAC, 79-89.

Stalman, Andy (2014). Brandoffon: el branding del futuro. Barcelona: Grupo Planeta. Statt, Nick (2017). Netflix plans to spend $\$ 8$ billion to make its library 50 percent original by 2018. En: https://www.theverge.com/2017/10/16/16486436/netflix-original-content-8-billion-dollars-anime-films (Consultado el 18-07-2019). 
Summers, Jim; Brecht, Tim; Eager, Derek y Gutarin, Alex (2016). Characterizing the workload of a Netflix streaming video server. En: IEEE International Symposium on Workload Characterization. Piscataway: IEEE Computer Society, 1-12.

Thompson, Nicholas (2003). Netflix's Patent May Reshape DVD-Rental Market. En: http://www.nytimes.com/2003/06/26/business/technology-netflix-s-patent-mayreshape-dvd-rental-market.html (Consultado el 18-07-2019).

Tucker, Matt (2015). La historia de Netflix y el futuro de la televisión. En: http:// www.elrincondemarketing.es/la-historia-de-netflix-y-el-futuro-de-la-television. (Consultado el 18-07-2019).

Vargas, Paulina (2015). Netflix refines responsive design with "The Stack". En: https://webdesignledger.com/netflix-refines-responsive-design-with-the-stack/ (Consultado el 18-07-2019).

Velilla, Javier (2010). Branding. Tendencias y retos en la comunicación de marca. Barcelona: UOC.

Whipps, Kevin (2016). Netflix's new Brand Identity might be the best we've seen all year. En: https://creativemarket.com/blog/netflixs-new-brand-identity-might-bethe-best-weve-seen-all-year (Consultado el 18-07-2019). 
